\title{
A continuous minimax problem for calculating minimum norm polynomial interpolation points on the sphere
}

\author{
Robert S. Womersley*
}

(Received 7 August 2000)

\begin{abstract}
This paper considers the calculation of the minimum norm points for polynomial interpolation over the sphere $S^{2} \subset \mathrm{R}^{3}$. The norm of the interpolation operator $\Lambda_{n}$, considered as a map from $C\left(S^{2}\right)$ to $C\left(S^{2}\right)$, is given by $\left\|\Lambda_{n}\right\|=\max _{x \in S^{2}}\left\|B^{-1} \mathbf{b}(x)\right\|_{1}$, where the nonsingular matrix $B$ and vector $\mathbf{b}$ are determined by the fundamental
\end{abstract}

*School of Mathematics, University of New South Wales, Sydney 2052, Australia. mailto:R.Womersley@unsw.edu.au

${ }^{0}$ See http: //anziamj . austms .org. au/V42/CTAC99/Wome for this article and ancillary services, (c) Austral. Mathematical Soc. 2000. Published 27 Nov 2000. 
system of points $x_{j} \in S^{2}, j=1, \ldots, d_{n}$. The problem is to choose the fundamental system to minimise $\left\|\Lambda_{n}\right\|$.

Algorithms for solving this continuous minimax problem must be able to handle many local maxima close to the global maximum, and local maxima which lie close to each other along ridges. A first order dual algorithm is used to find a spherical parametrisation of a normalised fundamental system. The results suggest that for these points the growth in $\left\|\Lambda_{n}\right\|$, for $n<30$, is less than $c_{0}+c_{1} n$, where $c_{0} \approx 1.8$ and $c_{1} \approx 0.7$.

\section{Contents}

1 Introduction

C1538

2 Reproducing kernel basis

C1541

3 Parametrisation and Derivatives

C1543

4 Algorithms

C1546

5 Results

C1552

References

C1554 


\section{Introduction}

This paper considers the use of a continuous minimax problem to find the minimum norm polynomial interpolation points on the unit sphere $S^{2} \subseteq \mathrm{R}^{3}$. Although much of the discussion extends to the sphere $S^{r-1}$ in $\mathrm{R}^{r}$ (see [15] for example), this paper only deals with $r=3$. Let $\mathrm{P}_{n}$ denote the space of all spherical polynomials of degree at most $n$ (i.e. the space of all polynomials in 3 variables restricted to $S^{2}$ ). Using the usual spherical polar coordinates $\theta \in[0, \pi]$ and $\phi \in[0,2 \pi)$, a common basis for $\mathrm{P}_{n}$ is the spherical harmonics [5]

$$
Y_{\ell k}=c_{\ell} P_{\ell}^{|k|}(\cos \theta) \cos (|m| \phi), \quad \ell=0, \ldots, n \text { and } k=-\ell, \ldots, \ell,
$$

where $c_{\ell}$ are appropriate normalisation constants and $P_{\ell}^{|k|}$ are the associated Legendre functions. The dimension of the space $\mathrm{P}_{n}$ is $d_{n}=(n+1)^{2}$.

The polynomial interpolant $\Lambda_{n} f$ coincides with a given continuous function $f$ at a prescribed set of points $\left\{x_{1}, \ldots, x_{d_{n}}\right\} \subseteq S^{2}$. A set of points $\left\{x_{1}, \ldots, x_{d_{n}}\right\} \subseteq S^{2}$ is a possible set of interpolation points for the space $\mathrm{P}_{n}$ if and only if it is a fundamental system, that is the zero polynomial is the only member of $\mathrm{P}_{n}$ that vanishes at each point $x_{j}, j=1, \ldots, d_{n}$.

The question is, for fixed $n$, to find $d_{n}=(n+1)^{2}$ points on $S^{2}$ so that polynomial interpolation is a good approximation. Fliege and Maier [2] suggest choosing the points to minimise the potential energy

$$
\rho\left(x_{1}, \ldots, x_{d_{n}}\right)=\sum_{i=1}^{d_{n}} \sum_{j=i+1}^{d_{n}} \frac{1}{\left\|x_{i}-x_{j}\right\|_{2}} .
$$


Another possible criterion is the mesh norm

$$
h=\max _{x \in S^{2}} \min _{j=1, \ldots, d_{n}} \operatorname{dist}\left(x, x_{j}\right), \quad \operatorname{dist}(x, y)=\cos ^{-1}\left(x^{T} y\right),
$$

which gives the maximum great circle distance from the closest point of $\left\{x_{1}, \ldots, x_{d_{n}}\right\}$. These criteria are primarily concerned with the geometric distribution of the points $\left\{x_{1}, \ldots, x_{d_{n}}\right\}$ on the sphere $S^{2}$, and are not directly related to the problem of polynomial interpolation.

The norm of the polynomial interpolation operator, as a map from $C\left(S^{2}\right)$ to $C\left(S^{2}\right)$ is

$$
\left\|\Lambda_{n}\right\|=\sup _{f \in C, f \neq 0} \frac{\left\|\Lambda_{n} f\right\|_{\infty}}{\|f\|_{\infty}} .
$$

As $\Lambda_{n}$ is a projection onto $\mathrm{P}_{n}$, i.e. $\Lambda_{n}$ is linear and $\Lambda_{n}^{2}=\Lambda_{n}$,

$$
\left\|\Lambda_{n} f-f\right\|_{\infty} \leq\left(1+\left\|\Lambda_{n}\right\|\right) E_{n}(f),
$$

where $E_{n}(f)=\inf _{p \in \mathrm{P}_{n}}\|f-p\|_{\infty}$ is the error of best uniform approximation. Because of its role in the upper bound (3) we use the Lebesgue constant $\left\|\Lambda_{n}\right\|$ as the criterion for selecting the interpolation points.

Let $b_{i} \in \mathrm{P}_{n}$ for $i=1, \ldots, d_{n}$ be a basis for $\mathrm{P}_{n}$, and define the vector valued function $\mathbf{b}: S^{2} \rightarrow \mathrm{R}^{d_{n}}$ by $\mathbf{b}(x)=\left[b_{1}(x) \cdots b_{d_{n}}(x)\right]^{T}$ and the interpolation matrix $B$ by

$$
B=\left[\mathbf{b}\left(x_{1}\right) \cdots \mathbf{b}\left(x_{d_{n}}\right)\right] \text { i.e. } B_{i j}=b_{i}\left(x_{j}\right) \quad i, j=1, \ldots, d_{n} .
$$


The matrix $B$ is nonsingular if and only if the set of points $\left\{x_{1}, \ldots, x_{d_{n}}\right\}$ is a fundamental system. The norm of the interpolation operator is then

$$
\left\|\Lambda_{n}\right\|=\max _{x \in S^{r-1}}\left\|B^{-1} \mathbf{b}(x)\right\|_{1} .
$$

The value of $\left\|\Lambda_{n}\right\|$ depends on the fundamental system $\left\{x_{1}, \ldots, x_{d_{n}}\right\}$. The norm $\left\|\Lambda_{n}\right\|$ can be made arbitrarily large if the fundamental system is badly chosen. The interesting question is how small $\left\|\Lambda_{n}\right\|$ can be made by a good choice of fundamental system.

Reimer $[10,11]$ has shown that an extremal fundamental system, i.e. a fundamental system which maximises $|\operatorname{det}(B)|$, has $\left\|\Lambda_{n}\right\| \leq d_{n}=(n+1)^{2}$. This bound is quite pessimistic. Another bound given by Reimer [10] is

$$
\left\|\Lambda_{n}\right\| \leq(n+1)\left(\frac{\lambda_{\mathrm{avg}}}{\lambda_{\min }}\right)^{1 / 2},
$$

where $\lambda_{\text {avg }}$ and $\lambda_{\min }$ are the average and minimum eigenvalues of the positivedefinite Gram matrix $G$ determined by the fundamental system using the reproducing kernel basis (see Section 2). The ratio $\lambda_{\text {avg }} / \lambda_{\min } \geq 1$ depends on the choice of points $\left\{x_{1}, \ldots, x_{d_{n}}\right\}$, and, less obviously (Reimer [10]), $\lambda_{\text {avg }} / \lambda_{\min }>1$ for $n \geq 3$.

It is known that the optimal order of growth for the norm of a linear projection onto $S^{2}$ is $O\left(n^{1 / 2}\right)$, which is achieved by the $L_{2}$-orthogonal projection and hyperinterpolation $[12,13]$. Several criteria for choosing a good set of 
polynomial interpolation points on the sphere are discussed in [15], as well as comparisons with some non-polynomial approximations.

This paper concentrates on the use of a continuous minimax algorithm to find a fundamental system which minimises the norm $\left\|\Lambda_{n}\right\|$ of the interpolation operator. For a background on continuous minimax problems, or their equivalent formulation as semi-infinite programming problems, see [4, 6, 9]. A significant aspect is the parametrisation of the fundamental system and the calculation of derivatives with respect to this parametrisation. These are considered in Section 3, after Section 2 gives details of the reproducing kernel basis used. Section 4 discusses the continuous minimax algorithm, with results given in Section 5.

\section{$2 \quad$ Reproducing kernel basis}

This section outlines a simple representation [13] of $\Lambda_{n} f$ which avoids explicit computation of spherical harmonics based on the reproducing kernel (Reimer [10])

$$
G_{n}(x, y):=\sum_{\ell=0}^{n} \sum_{k=-\ell}^{\ell} Y_{\ell k}(x) Y_{\ell k}(y), \quad x, y \in S^{2} .
$$


The Addition Theorem [5] for spherical harmonics shows that $G_{n}(x, y)$ is bizonal, depending only on the angle between $x$ and $y$, so

$$
G_{n}(x, y)=\tilde{G}_{n}\left(x^{T} y\right) .
$$

In particular, for $S^{2}$

$$
\tilde{G}_{n}(z)=\frac{1}{4 \pi} \sum_{\ell=0}^{n}(2 \ell+1) P_{\ell}(z),
$$

where $P_{\ell}(\cdot)$ is the usual Legendre polynomial. This result can be written in closed form [3] $\tilde{G}_{n}(z)=\frac{n+1}{4 \pi} P_{n}^{(1,0)}(z)$ using the Jacobi polynomial $P_{n}^{(1,0)}$ of degree $n$ corresponding to the weight function $(1-z)$ on $[-1,1]$ (see Szegö [14]).

Using the standard recurrence for Legendre polynomials $\tilde{G}_{n}(z)$ can be evaluated by

$$
\begin{aligned}
& P_{0}(z)=1, \quad P_{1}(z)=z, \quad \tilde{G}_{1}(z)=P_{0}(z)+3 P_{1}(z), \\
& P_{n}(z)=\frac{2 n-1}{n} P_{n-1}(z)-\frac{n-1}{n} P_{n-2}(z), \\
& \tilde{G}_{n}(z)=\tilde{G}_{n-1}(z)+(2 n+1) P_{n}(z),
\end{aligned}
$$

for $n \geq 2$ and $z \in[-1,1]$. The derivative $\tilde{G}_{n}^{\prime}(z)$ can also easily be calculated using this recurrence.

For each point $x_{j}$ of the fundamental system $\left\{x_{1}, \ldots, x_{d_{n}}\right\}$ define the kernel polynomials $g_{j} \in \mathrm{P}_{n}$ with axis $x_{j}$ by

$$
g_{j}(x)=G_{n}\left(x, x_{j}\right)=\tilde{G}_{n}\left(x^{T} x_{j}\right), \quad j=1, \ldots, d_{n} .
$$


Define the vector valued function $\mathbf{g}: S^{2} \rightarrow \mathrm{R}^{d_{n}}$ by $\mathbf{g}(x)^{T}=\left[g_{1}(x) \cdots g_{d_{n}}(x)\right]$. The corresponding basis matrix is

$$
G=\left[\mathbf{g}\left(x_{1}\right) \cdots \mathbf{g}\left(x_{d_{n}}\right)\right], \quad \text { i.e. } G_{i j}=\tilde{G}_{n}\left(x_{i}^{T} x_{j}\right) \text { for } i, j=1, \ldots, d_{n} .
$$

Then the weights $\mathbf{w}$ such that $\left(\Lambda_{n} f\right)(x)=\mathbf{w}^{T} \mathbf{g}(x)$ are given by the linear system $G \mathbf{w}=\mathbf{f}$, where $\mathbf{f}=\left[f\left(x_{1}\right) \cdots f\left(x_{d_{n}}\right)\right]^{T}$. Using this reproducing kernel basis the norm of the interpolation operator is

$$
\left\|\Lambda_{n}\right\|=\max _{x \in S^{2}}\left\|G^{-1} \mathbf{g}(x)\right\|_{1} .
$$

\section{Parametrisation and Derivatives}

The problem is to find a fundamental system of interpolation points $X=$ $\left\{x_{1}, \ldots, x_{d_{n}}\right\}$ to minimise the norm of the interpolation operator. Making the dependence of $G$ and $\mathbf{g}$ on the fundamental system $X$ explicit, the problem is

$$
\min _{X} \max _{x \in S^{2}}\left\|G(X)^{-1} \mathbf{g}(X ; x)\right\|_{1} .
$$

This section considers the parametrisation of the fundamental system $X$ and derivatives of $G(X), \mathbf{g}(X ; x)$ and $\left\|\Lambda_{n}(X)\right\|$ with respect to a spherical parametrisation of the fundamental system $X$.

In general the definition of continuously differentiable functions over $S^{2}$ is complicated, requiring the division of $S^{2}$ into pieces to obtain local parametri- 
sations in 2D Euclidean space and then requiring any continuously differentiable function to have continuous derivatives with respect to each local coordinate system (see $[3, \S 3]$ for example).

A critical property is that $G(X), \mathbf{g}(X ; x)$ and $\left\|\Lambda_{n}(X)\right\|$ are invariant under an arbitrary rotation, that is for any orthogonal $Q \in \mathrm{R}^{3 \times 3}$,

$$
G(Q X)=G(X), \quad \mathbf{g}(Q X ; Q x)=\mathbf{g}(X ; x), \text { and }\left\|\Lambda_{n}(Q X)\right\|=\left\|\Lambda_{n}(X)\right\| .
$$

It is also convenient to work with a spherical polar representation of $x \in S^{2}, t=[\theta \phi]^{T} \in T=[0, \pi] \times[0,2 \pi)$ so

$$
x=\left[\begin{array}{lll}
\sin t_{1} \cos t_{2} & \sin t_{1} \sin t_{2} & \cos t_{1}
\end{array}\right]^{T} .
$$

As the spherical polar coordinates are oriented along the third coordinate axis, the rotational invariance is used, as in [2], to fix the first point $x_{1}$ of the fundamental system at the north pole $\left(\theta_{1}=0, \phi_{1}\right.$ irrelevant) and the second point $x_{2}$ on the prime meridian $\left(\phi_{2}=0\right)$. The gives the $p=2 d_{n}-3$ parameters

$$
\theta_{i} \in[0, \pi], \quad i=2, \ldots, d_{n}, \quad \phi_{j} \in[0,2 \pi), \quad j=3, \ldots, d_{n} .
$$

The parameters specifying the fundamental system are grouped in the vector

$$
s=\left[\theta_{2}, \ldots, \theta_{d_{n}}, \phi_{3}, \ldots, \phi_{d_{n}}\right]^{T} \in \mathrm{R}^{p}
$$


Thus for $n=29$ there are $d_{n}=900$ interpolation points specified by $p=$ $2 d_{n}-3=1797$ parameters.

In fact to allow the straightforward differentiation of functions of $t$ and $s$, these variables are restricted to regions of the sphere $S^{2}$ excluding small caps around the north and south poles. One interpolation point is fixed at the north pole, and hence does not appear explicitly as a minimisation variable. This, together with an explicit consideration of the south pole, avoids the singularity associated with $\theta$ becoming negative along an $\operatorname{arc}$ through $\theta=0$, or $\theta$ exceeding $\pi$ on an arc through $\theta=\pi$, when $\phi$ changes by $\pi$.

It should be noted that any two points in a fundamental system can be swapped, along paths on which they do not coincide. The swap does not change the value of $\left\|\Lambda_{n}\right\|$, but along this path the matrix $G$ is singular, so $\left\|\Lambda_{n}\right\|$ is infinite. Thus in the variables $s \in \mathrm{R}^{p}$ there are many local minimisers, separated by infinitely high walls. The optimisation procedure will only be able to produce a fundamental system which is an approximate local minimiser of $\left\|\Lambda_{n}\right\|$.

As the spherical polar coordinates are varied it is better not to impose the bounds in an optimisation procedure as points may become artificially stuck against one of the bounds, in particular the bounds on $\phi$. The final values can be be mapped to $[0, \pi] \times[0,2 \pi)$ using the $2 \pi$ periodicity in $\theta$ and $\phi$, along with the fact that $[\theta+\pi, \phi]^{T}$ corresponds to $[\theta, \phi \pm \pi]$, with the sign chosen so that $\phi \pm \pi \in[0,2 \pi)$. If these transformations are implemented at each stage, they pose particular difficulties for methods which use differences 
in points, for example quasi-Newton methods [1].

\section{Algorithms}

From now on only the spherical polar representations $t \in T$ of $x \in S^{2}$ and $s \in \mathrm{R}^{p}$, where $p=2 d_{n}-3$, of a fundamental system $X$ are considered. The continuous minimax problem

$$
\min _{s \in \mathrm{R}^{p}} \max _{t \in T}\|\mathbf{r}(s ; t)\|_{1}
$$

where $\mathbf{r}: \mathrm{R}^{p} \times T \rightarrow \mathrm{R}^{d_{n}}, \mathbf{r}(s ; t)=G(s)^{-1} \mathbf{g}(s ; t)$ is equivalent to the semiinfinite nonlinear programming problem

$$
\begin{array}{ll}
\min _{s \in \mathrm{R}^{p}, \nu \in \mathrm{R}} & \nu \\
\text { subject to } & \nu \geq\|\mathbf{r}(s ; t)\|_{1} \text { for all } t \in T .
\end{array}
$$

Algorithms for continuous minimax problems typically convert the inner maximisation in (13) into a maximum over a finite set (or equivalently the semi-infinite constraint in (14) into a finite set of constraints) either by working with meshes for $T$ which can be made finer and finer (see $[6,16]$ for example), or by finding all the local maxima which achieve the global maximum (see $[4,6,9,8,7]$ for example). 
I-berpola fion nem function, Mrimum rem poirta, $n=15 d_{n}=256$
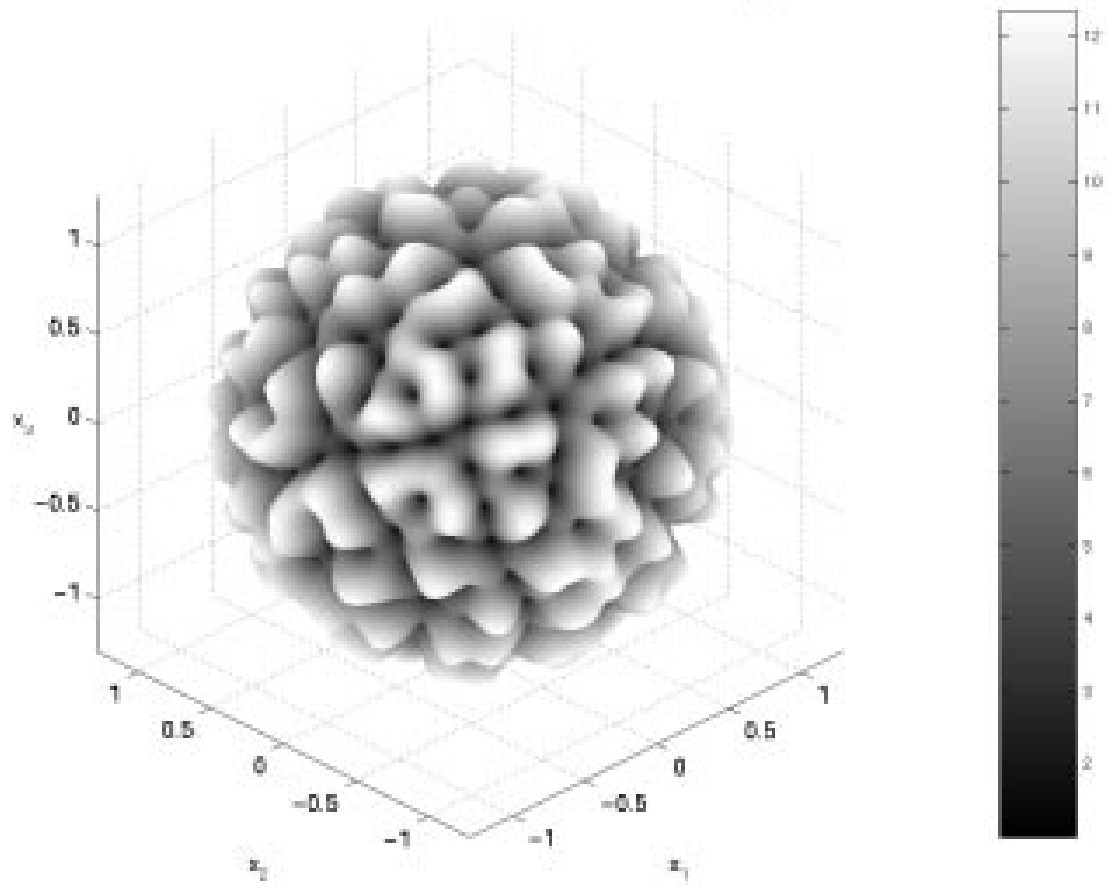

FiguRE 1: Interpolation norm function $\left\|G^{-1} \mathbf{g}(x)\right\|_{1}$ for $n=15$ 
First consider the inner maximisation in (13) for a fixed $s$. Let

$$
\psi(s)=\max _{t \in T} \sum_{i=1}^{d_{n}}\left|r_{i}(s ; t)\right| .
$$

If $r_{i}(s ; t) \neq 0$ for all $i=1, \ldots, d_{n}$ then $\|\mathbf{r}(s ; t)\|_{1}$ is a smooth function of $t$ for $t$ in regions excluding caps around the north and south poles. As $t$ is chosen to maximise $\|\mathbf{r}(s ; t)\|_{1}$ this might be expected to be the case. However the outer minimisation w.r.t. the parametrisation $s$ of the fundamental system may force one or more components of $r_{i}(s ; t)$ to be zero.

A necessary condition for a point $\bar{t}(s) \in(\epsilon, \pi-\epsilon) \times R$, for $\epsilon>0$ small, to be a local maximum of $\|\mathbf{r}(s ; t)\|_{1}$ is that

$$
0 \in \partial_{t}\|r(s ; \bar{t}(s))\|_{1}=\left\{u \in \mathrm{R}^{2}: u=\sigma(s) G(s)^{-1} \nabla_{t} \mathbf{g}(s, \bar{t}(s))\right\},
$$

where, for $i=1, \ldots, d_{n}$,

$$
e_{i}^{T} \sigma(s) \in\left\{\begin{array}{cl}
1 & \text { if } r_{i}(s, \bar{t}(s))>0 \\
{[-1,1]} & \text { if } r_{i}(s, \bar{t}(s))=0 \\
-1 & \text { if } r_{i}(s, \bar{t}(s))<0
\end{array}\right.
$$

and $e_{i}$ is the $i$ th unit vector in $\mathrm{R}^{d_{n}}$.

Efficient methods for solving (13) require all local maxima close to the global maximum. Moreover as the norm is minimised many points are expected to achieve the global maximum. For instance for $n=15, d_{n}=256$, 


\section{Interpolation norm function, Minimum norm points, $n=15, d_{n}=256$}

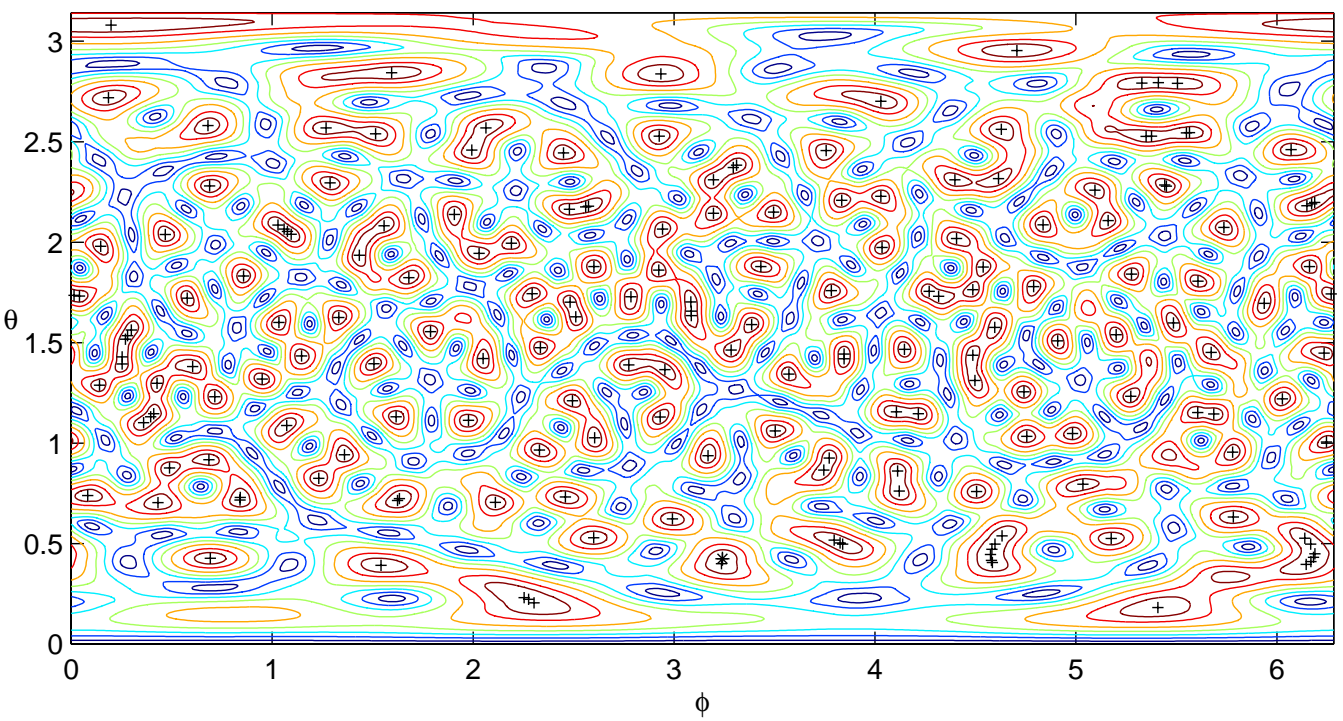

Figure 2: Contours of $\left\|G^{-1} \mathbf{g}(t)\right\|_{1}$ for $n=15$ 
the interpolation norm function $\left\|G^{-1} \mathbf{g}(t)\right\|_{1}$, is plotted in Figures 1 and 2 at the fundamental system of points obtained by minimising the norm. Figure 1 plots the points $\left(1+\xi /\left(f_{u}-f_{l}\right)\left(f(x)-f_{l}\right)\right) x$, for $x \in S^{2}$, where $f_{l}=\min _{x \in S^{2}} f(x), f_{u}=\max _{x \in S^{2}} f(x)$ and $\xi=0.3$ is a scaling parameter. Figure 2 plots the contours of $\left\|G^{-1} \mathbf{g}(t)\right\|_{1}$, with local maximisers marked with a + and a global maximiser with a $*$.

The strategy adopted was to calculate $\left\|G^{-1} \mathbf{g}(t)\right\|_{1}$ on a grid of points over $T$, explicitly including the north and south poles, identifying all grid local maxima within $\beta \epsilon, \beta>1$, of the global maximum over the grid, finding local maxima accurately from each of these starting points, taking the largest of these as $\left\|\Lambda_{n}\right\|$ and using all distinct local maxima within $\epsilon$ of the largest in the search direction subproblems. The grid may have to be refined, as it is a common occurrence in these problems for local maxima to occur along ridges (see Figures 1 and 2). A 201 by 402 equally spaced grid of $T$ lead to the identification of 177 local maxima within $1 \%$ of the global maximum for the example in Figure 1. An open question is whether a true global minimum norm fundamental system, as distinct from an approximate local minimiser, has curves in $T$ along which the inner maximum is achieved.

Assume there are $L(s)$ distinct local maximisers $y_{l}(s) \in S^{2}$ of $\left\|G^{-1} \mathbf{g}(t)\right\|$ within $\epsilon$ of the global maximum $\psi(s)$. Let $t_{\ell}(s)$ be the spherical polar representation of $y_{\ell}(s)$ and let $\psi_{\ell}(s)=\left\|\mathbf{r}\left(s, t_{\ell}(s)\right)\right\|_{1}$. The outer optimisation procedure requires the derivatives w.r.t. the spherical parametrisation $s$. Formally, assuming $G(s)$ is positive definite in a neighbourhood of $s$,

$$
\partial_{s}\left\|\mathbf{r}\left(s, t_{\ell}(s)\right)\right\|_{1}=\sigma_{\ell}(s)^{T} G(s)^{-1}\left[-\nabla_{s} G(s) G(s)^{-1} \mathbf{g}\left(s, t_{\ell}(s)\right)+\right.
$$




$$
\left.\nabla_{s} \mathbf{g}\left(s, t_{\ell}(s)\right)+\nabla_{t} \mathbf{g}\left(s, t_{\ell}(s)\right) \nabla_{s} t_{\ell}(s)\right] .
$$

If the inner maximisation is smooth at $t_{\ell}(s)$ then $\sigma_{\ell}(s)$ is uniquely determined by (17). If it is nonsmooth then $\sigma_{\ell}(s)$ is determined by (17) and (16). In either case, $\sigma_{\ell}(s)^{T} G(s)^{-1} \nabla_{t} \mathbf{g}\left(s, t_{\ell}(s)\right)=0$, so the last term in (18) vanishes. Hence derivatives of $t_{\ell}(s)$ w.r.t. $s$ are not needed in first order information.

Let $v_{\ell}(s) \in \partial_{s}\left\|r\left(s, t_{\ell}(s)\right)\right\|_{1}$. The primal subproblem to determine a search direction $h \in \mathrm{R}^{p}$ is

$$
\begin{array}{cl}
\min _{h \in \mathrm{R}^{p}, \nu \in \mathrm{R}} & \nu+\frac{\delta}{2}\|h\|_{2}^{2} \\
\text { subject to } & \nu \geq \psi_{\ell}(s)+h^{T} v_{\ell}(s) \quad \ell=1, \ldots, L(s)
\end{array}
$$

which is a quadratic programming problem in $p+1$ variables with $L(s)$ constraints. A piecewise linear line search can be used to find an approximate minimiser $\bar{\alpha}$ of $\psi(s+\alpha h)$, giving the next iterate $\bar{s}=s+\bar{\alpha} h$. Alternatively a trust region $\|h\|_{\infty} \leq \eta$ can be added to the search direction subproblem (19) with the trust region radius being updated by rules based on the agreement between $\psi(s+h)$ and the predicted value $\bar{\nu}$ (see [1] for example).

The corresponding dual search direction subproblem is

$$
\min _{\left[\begin{array}{c}
u^{0} \\
u
\end{array}\right] \in \mathcal{G}(s)} u^{0}+\frac{\delta}{2}\|u\|_{2}^{2},
$$

where

$$
\mathcal{G}(s)=\underset{\ell=1, \ldots, L(s)}{\operatorname{conv}}\left\{\left[\begin{array}{c}
\psi(s)-\psi_{\ell}(s) \\
v_{\ell}(s)
\end{array}\right]\right\} .
$$


The dual search direction is $h=-\bar{u}$ where $\left[\bar{u}^{0} \bar{u}\right]^{T}$ solves the subproblem (20). Expressing

$$
\mathcal{G}(s)=\left\{\sum_{i=1}^{L(s)} \lambda_{i}\left[\begin{array}{c}
\psi(s)-\psi_{\ell}(s) \\
v_{\ell}(s)
\end{array}\right]: \sum_{i=1}^{L(s)} \lambda_{i}=1, \lambda_{i} \geq 0, i=1, \ldots, L(s)\right\},
$$

shows that (20) is also a quadratic programming problem, but with $L(s)$ variables and a single equality constraint. Because the number $L(s)$ of dual problem is typically only a few hundred for $n<30$, while the number of primal variables $p$ is up to 1797 , the dual subproblem was used to determine the search direction. The parameter $\delta$ can be estimated from information obtained in the line search [6].

\section{Results}

The dual algorithm outlined in Section 4 was used to find a fundamental system that is an approximate local minimiser of $\left\|\Lambda_{n}\right\|$. As discussed in Section 3 there are likely to be many local minimisers, so the choice of starting point is critical. The algorithm was started from the eigenvalue points [15], which were obtained by choosing the fundamental system to maximise $\lambda_{\min }$, and hence to minimise the bound (6) on $\left\|\Lambda_{n}\right\|$. The maximum determinant points were obtained by choosing the fundamental system to maximise $\operatorname{det} G$. The potential energy points are those of Fliege and Maier [2]. 

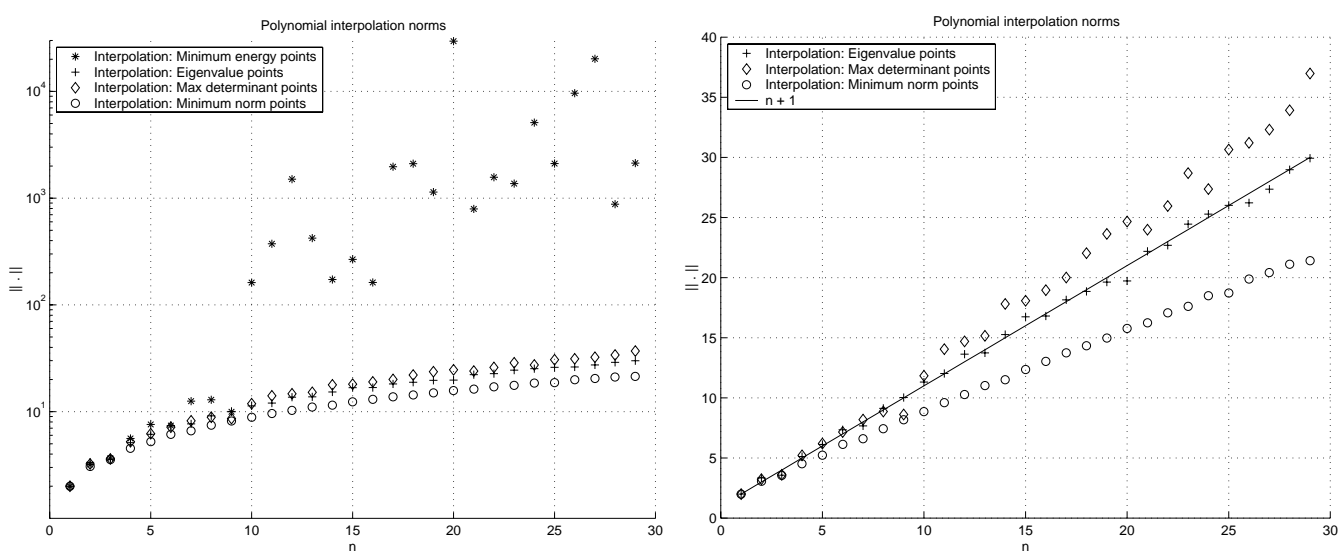

FigURE 3: Norms of the polynomial interpolation operators 
The norms $\left\|\Lambda_{n}\right\|$ are plotted in Figure 3 as functions of $n$. A notable feature of the results in Figure 3 is that the potential energy points are not very good and that the minimum norm points have smaller norm than the maximum determinant and eigenvalue points. Numerically $\left\|\Lambda_{n}\right\| \approx 1.8+0.7 n$ for the minimum norm points. A detailed comparison of different point sets and related approximations can be found in [15]. It remains an open question what the rate of growth of $\left\|\Lambda_{n}\right\|$ (as a function of $n$ ) is for fundamental systems achieving the global minimum, and if these minimum norm points can be characterised in terms of the zeros of an orthogonal polynomial. Various point sets and plots of different criteria are available from http://www. maths/unsw. edu.au/ ${ }^{\text {rsw }} /$ Sphere.

Acknowledgements: This paper stems from ongoing work with Ian Sloan, whose stimulating comments are gratefully acknowledged. The work is supported by the Australian Research Council with programming support from Dave Dowsett.

\section{References}

[1] Roger Fletcher. Practical Methods of Optimization. John Wiley, Chichester and New York, second edition, 1987. C1546, C1551 
[2] Jörg Fliege and Ulrike Maier. The distribution of points on the sphere and corresponding cubature formulae. IMA J. Num. Anal., 19(2):317-334, 1999.

http://www . mathematik . uni-dortmund.de/lsx/fliege/nodes.html. C1538, C1544, C1552

[3] M. Ganesh, Ivan Graham, and J. Sivaloganathan. A pseudospectral three-dimensional boundary integral method applied to a nonlinear model problem from finite elasticity. SIAM J. Numer. Anal., 31(5):1378-1414, 1994. C1542, C1544

[4] Rainer Hettich and Kenneth O. Kortanek. Semi-infinite programming: theory, methods and applications. SIAM Review, 35(3):380-429, 1993. C1541, C1546

[5] C. Müller. Spherical Harmonics, volume 17 of Lecture Notes in Mathematics. Springer Verlag, Berlin, New-York, 1966. C1538, C1542

[6] Elijah Polak. Optimization: Algorithms and Consistent Approximations. Springer-Verlag, New York, 1997. C1541, C1546, C1546, C1552

[7] C. J. Price and I. D. Coope. Numerical experiments in semi-infinite programming. Computational Optimization and Applications, 6(2):169-189, 1996. C1546

[8] C. J. Price and Ian Coope. An exact penalty function algorithm for semi-infinite programmes. BIT, 30(4):723-734, 1990. C1546 
[9] Rembert Reemtsen and Jan-J. Rückman. Semi-infinite Programming, volume 25 of Noncovex Optimization and its Applications. Kluwer Academic Publishers, Boston, MA, 1998. C1541, C1546

[10] Manfred Reimer. Constructive Theory of Multivariate Functions. BI Wissenschaftsverlag, Mannheim, Wien, Zürich, 1990. C1540, C1540, C1540, C1541

[11] Manfred Reimer. Quadrature rules for the surface integral of the unit sphere based on extremal fundamental systems. Mathematische Nachrichten, 169:235-241, 1994. C1540

[12] Ian H. Sloan. Polynomial interpolation and hyperinterpolation over general regions. J. Approx. Theory, 83:238-254, 1995. C1540

[13] Ian H. Sloan and Robert S. Womersley. Constructive polynomial approximation on the sphere. Journal of Approximation Theory, 103:91-118, 2000. C1540, C1541

[14] G. Szegö. Orthogonal Polynomials, volume 23 of American Mathematical Society Colloquium Publications. American Mathematical Society, Providence, Rhode Island, 4th edition, 1975. C1542

[15] Robert S. Womersley and Ian H. Sloan. How good can polynomial interpolation on the sphere be? Applied Mathematics Report AMR99/25, School of Mathematics, University of New South Wales, Sydney, Australia, 1999. C1538, C1541, C1552, C1554 
[16] Jian L. Zhou and André L. Tits. An SQP algorithm for finely discretized continuous minimax problems and other minimax problems with many objective functions. SIAM J. Optim., 6(2):461-487, 1996. C1546 